\title{
LAS ENCUESTAS ELECTORALES EN LOS DIARIOS ARGENTINOS
}

\author{
María Eugenia Tesio y Belén Amadeo 2
}

\section{Resumen}

Los medios de comunicación suelen mostrarse muy interesados por los datos que las encuestas brindan a la hora de cubrir las campañas electorales y los diarios argentinos no son una excepción. En este trabajo analizaremos qué uso hicieron los principales periódicos nacionales de las encuestas como herramientas cuantitativas durante la cobertura de la campaña presidencial de 2015. ¿Qué aspectos de la contienda electoral prefieren fundamentar con datos duros?, ¿Se muestran las fichas técnicas?, ¿Cómo se las muestra? Buscamos comprender qué hábitos de cobertura tienen

\footnotetext{
${ }^{1}$ María Eugenia Tesio: Universidad de Buenos Aires. Facultad de Ciencias Sociales, Av. Figueroa Alcorta 3200 CABA CP 1425. Argentina Tel: 005491159297754. Mail:marutesio@gmail.com.

Doctora en Ciencias Sociales (UBA). Magíster en Historia (UTDT). Posgrado en Gobierno de la Universidad de Harvard, Estados Unidos. Licenciada en Ciencia Política (UBA). Profesora de la Facultad de Ciencias Sociales (UBA), de la Universidad CEMA y Flacso. Investigadora y coordinadora de grupos de investigación de la UBA y en el Instituto de Sociologia Politica de la Academia Nacional de Ciencias Morales y Políticas. Principales publicaciones: coautora de los libros Elecciones 99. Análisis de los resultados (1999) y Argentina en las Urnas 1916-1994 (1995) ambos con el doctor Rosendo Fraga; diversos artículos presentados y publicados en congresos nacionales e internacionales sobre temas electorales en Argentina y Latinoamérica.

${ }^{2}$ Belén Amadeo: Universidad de Buenos Aires. Facultad de Ciencias Sociales, Rodríguez Peña 1456 CABA CP 1021. Argentina Tel: 005491136033600. Mail:belenamadeo@gmail.com.

Doctora en Comunicación Pública (Universidad de Navarra). Licenciada en Ciencia Política (UBA). Profesora de grado en las materias Opinión Pública (UBA), Comunicación Política (Universidad de Montevideo) y Sistemas Políticos Contemporáneos (Universidad de Montevideo). Profesora de Comunicación y Política en la Maestria en Gobierno (UBA), de Opinión Pública en la Maestría en Comunicación Política (Untref), de Comunicación Política en la Maestría de Comunicación Estratégica (Universidad de Montevideo). Ha dictado clases de maestria y doctorado en varias universidades públicas y privadas del país, de Colombia y de Ecuador. Es investigadora en la Universidad de Buenos Aires.
} 
los diarios La Nación, Clarín y Página 12 para trabajar con encuestas durante las campañas y si cumplieron con las pautas internacionales de calidad de publicación y tratamiento de encuestas establecidas por Esomar/Wapor.

Palabras clave: encuestas, elecciones, medios, ficha técnica, consultoras, opinión pública

\section{Las encuestas electorales en los diarios argentinos}

\section{Los criterios internacionales para la cobertura de las encuestas}

La relación entre los tres actores de la opinión pública, los dirigentes políticos, los medios de comunicación y los ciudadanos (Wolton, 1998), ha probado ser conflictiva y desigual. La importancia que cada uno de estos actores tiene y la que cree tener con respecto a los otros dos, el tipo de interacción que los caracteriza, la confianza que hay entre ellos, el papel que cada uno de los actores asume para sí mismo y las expectativas puestas en ellos afectan y se ven afectadas por la cultura política y la historia reciente de cada país.

En países democráticos con sistemas electorales tradicionales, esto se manifiesta de manera evidente durante los períodos de campañas previos a las elecciones. Si en esos períodos consideramos que la voz de los dirigentes es la comunicación electoral; la voz de los medios se manifiesta en sus páginas y la voz de la gente, en las encuestas, el análisis de los discursos de cada uno de los actores se hace relativamente sencillo de captar. No obstante, la voz de los ciudadanos no es tan clara. Son los dirigentes políticos o los medios de comunicación quienes contratan consultoras de opinión pública para que releven la opinión de la gente y solo algunas de ellas son transmitidas al público parcialmente y a través de escasos medios.

Esto no es nuevo. Alonso, Cabrera y Tesio (2009: 17) sostienen que "los expertos ven las dificultades que tienen los políticos y los medios para utilizar de manera apropiada la información que les brinda el instrumento [las encues- 
tas]. Del otro lado, los políticos le atribuyen una cuota importante de la responsabilidad al trabajo de los propios expertos. Y, en ambos casos, usan como chivo expiatorio a los medios, atribuyéndoles el mal manejo de los datos".

Ante la evidencia de que en los medios europeos y estadounidenses la difusión de las encuestas tampoco se daba de manera correcta, o por lo menos precisa, en 2006 la Sociedad Europea para la Investigación en Opinión y Marketing y la Asociación Mundial de Investigación en Opinión Pública (Esomar y Wapor, respectivamente, por sus siglas en inglés) delinearon una guía para sondeos de opinión y encuestas publicadas. Ambas instituciones, dedicadas esencialmente a la calidad de los estudios de opinión pública y al diseño y la cobertura de encuestas de opinión, vieron la necesidad de difundir a públicos no especializados cuáles son las características de una investigación de calidad y cómo debe publicarse para evitar confusiones en lectores no avezados en esas lides.

Una buena cobertura supone el respeto a una ética periodística básica que busca analizar la información de manera acabada y pone a disposición de los lectores todos los escenarios posibles basados en los análisis de datos concretos. Esta guía se encuentra traducida al castellano desde 2009.

Para que el público general tenga acceso a información imparcial y precisa en este campo, los medios de comunicación deben respetar una serie de disposiciones para la publicación de notas relacionadas con encuestas de opinión. En su artículo 3, la Guía Esomar/Wapor establece que las encuestas de opinión publicadas en un medio impreso deben estar acompañadas de una clara y distinguida aclaración de los siguientes puntos: "(a) el nombre de la organización de investigación que realiza el sondeo; (b) el universo efectivamente representado (es decir, quién fue entrevistado); (c) el tamaño de la muestra conseguida y su cobertura geográfica; (d) las fechas del trabajo de campo; (e) el método de muestreo utilizado (y en el caso de muestras com- 
pletamente aleatorias, la tasa de respuesta obtenida); (f) el método por el que se recogió la información (cara a cara, entrevista telefónica, panel de internet, etc.); (g) si se aplicó una ponderación para ajustar los resultados, y qué universo se utilizó para ello; (h) las preguntas realizadas que sean relevantes. Con el fin de evitar posibles ambigüedades se debe dar el texto de la pregunta a menos que se trate de una pregunta estándar ya familiar para la audiencia o se haya dado en un informe previamente publicado al que se hace referencia" (Esomar/Wapor, 2009, p. 14).

En este trabajo analizamos con cuánto rigor se transmite la información en la Argentina cuando los medios hablan sobre los sondeos o sobre el estado de la opinión de los ciudadanos con respecto a sus líderes, sus proyectos o sus partidos. ¿Se respetan las pautas de la guía Esomar/Wapor? Los periodistas argentinos consideran que su principal función social es la de formar a los ciudadanos (Amado y Bongiovanni, 2016). En este sentido, es interesante observar si el público efectivamente recibe datos claros, ciertos y fundamentados con información técnica que avale las conclusiones de los periodistas.

\section{Elecciones presidenciales y medios comprometidos}

El sistema argentino establece que el presidente se elige por doble vuelta electoral. Los artículos 97 y 98 de la Constitución Nacional prevén la realización de un balotaje en dos situaciones: cuando ninguno de los candidatos se alza con el $45 \%$ de los votos en primera vuelta o cuando ninguno supera el 40\% con una diferencia de más del 10\% respecto del que le sigue en número de sufragios. En 2015, la primera vuelta electoral tuvo lugar el 25 de octubre y la segunda, el 22 de noviembre. Todo el proceso electoral de ese año se dio en un contexto de altísima politización del debate público. Luego de doce años de gobierno kirchnerista, existía la genuina posibilidad de un cambio de signo político, lo que generaba ansiedad y elevados niveles de agresión en los discursos de propios y ajenos. 
Como siempre ocurre, los medios de comunicación argentinos reflejaron las características y las contradicciones de la sociedad a la que pertenecen. En el caso analizado, hubo una confrontación política clara entre la entonces presidenta Cristina Fernández y los partidos de la oposición; ambas partes tenían sus defensores y detractores en distintos medios. Entre los diarios, el más destacado defensor del gobierno era Página/12 y quienes apoyaban a la oposición eran Clarín y La Nación.

Clarín es el principal diario del país. Nació en 1945 y es el de mayor circulación en el mundo de habla hispana (Albornoz, 2006). Desde mediados de los años ochenta y con mayor intensidad durante la década de 1990, ha tenido un significativo crecimiento empresarial, a nivel horizontal, vertical y conglomeral (Becerra y Mastrini, 2009). Su gran nivel de tirada ha sido determinante en su capacidad de fijar la agenda de temas en el público y en otros medios.

Creado en 1870, La Nación es uno de los diarios más antiguos del país. Su línea editorial pone de manifiesto una preocupación por mantener "los principios del liberalismo bien entendido, polifacético" (Ulanovsky, 1997). En este sentido, si bien este matutino se caracterizó por la polifonía ideológica, nunca abandonó su filosofía liberal y tradicionalista.

En cuanto a Página 12, su historia y características son muy distintas de las de los otros dos diarios. Nació en mayo de 1987 representando un espacio político de izquierda que iba desde progresistas hasta independientes de izquierda. Su principal público se encuentra en los ámbitos políticos y universitarios, por lo que el nivel educativo del lector de Página 12 es el más alto de todos (Amadeo, 1999). Desde su origen y hasta comienzos del gobierno de Néstor Kirchner se caracterizó por ser fuertemente opositor a todos los gobiernos. Eso cambió durante el kirchnerismo, cuando el diario pasó a defender las banderas oficialistas. 


\section{Diseño de la investigación}

Este trabajo busca presentar la instancia exploratoria inicial de una investigación en la que se realizará un análisis de contenido ${ }^{3}$ cuyos resultados se contrastarán con entrevistas en profundidad a los redactores y editores de los diarios en cuestión. En esta primera etapa expondremos los supuestos sobre los que se sustenta la investigación, los inconvenientes que encontramos a la hora de elaborar el código de análisis para llevar adelante este análisis.

\subsection{El corpus}

El universo de análisis está formado por las notas publicadas en las secciones Política (o equivalentes) y Opinión de los diarios Clarín, La Nación y Página 12, entre el 20 de septiembre y el 25 de noviembre de 2015, que mencionaban el concepto de "encuesta". La fecha de inicio es la que dispone el Código Electoral Nacional como comienzo de la campaña de la primera vuelta electoral y la de finalización es el día en el que se realizó la segunda vuelta electoral (Ley 19.945, texto ordenado por decreto $\mathrm{N}^{\circ} 2135 / 83$ ).

\section{Cuadro 1.}

Cantidad de notas por medio elevado

\begin{tabular}{|l|c|c|c|c|}
\hline & Clarin & Página 12 & La Nación & Total \\
\hline $\begin{array}{l}\text { Cantidad de notas que } \\
\text { mencionan el término } \\
\text { encuesta/s }\end{array}$ & 30 & 43 & 157 & 230 \\
\hline $\begin{array}{l}\text { \% de notas que } \\
\text { mencionan el término } \\
\text { encuesta/s }\end{array}$ & $13 \%$ & $19 \%$ & $68 \%$ & $100 \%$ \\
\hline
\end{tabular}

Fuente: Elaboración propia.

En total, se recabaron 230 artículos que cubren o que simplemente mencionan las encuestas referidas a las elecciones nacionales (Ver Cuadro 1). Quedaron fuera de este aná-

\footnotetext{
${ }^{3}$ Para una exhaustiva descripción de las técnicas de análisis de contenido, ver K. Krippendorff, 1980; C. R. Hoffstetter, 1981 y J. J. Sánchez Aranda, 2005.
} 
lisis las encuestas sobre elecciones provinciales y sobre factores económicos o sociales también presentados por los medios en ese período. Esto nos permitió calcular hasta qué punto los medios fundamentan sus opiniones en encuestas validando esos datos de manera rigurosa. Una vez recabados los artículos, analizamos si seguían las pautas de cobertura internacionalmente acordadas por Esomar y Wapor para así evaluar la calidad del tratamiento que los medios de comunicación hicieron de las encuestas durante el período electoral.

Al universo de artículos recabados se lo cuantificó para poder establecer la relevancia noticiosa que tuvo este caso a lo largo del período analizado. Posteriormente, realizamos una inmersión cualitativa para la elaboración del libro de códigos (Igartua, 2006; Miller y Riechert, 1994 y 2001).

\subsection{El libro de códigos}

Mediante el análisis de algunos indicadores se procura vislumbrar si el tratamiento de la prensa contribuyó a dar relevancia a las notas en las que se hablaba de encuestas o no. Se decidió analizar todos los artículos de las secciones de Opinión y de Política o similares en los cuales se nombrara el término "encuesta". Para ello se recurrió a los buscadores de la versión digital de los diarios. Una vez listados los artículos que contenian esas menciones, se descartaron aquellos que aludian a las elecciones provinciales o a encuestas de corte social o económico. En tercer lugar, se contrastó este listado con las ediciones en papel de esos diarios y se vio que la edición impresa publicada digitalmente y la edición papel de Página 12 y Clarín eran idénticas. Otro fue el caso de La Nación, cuyo buscador listó las notas del diario en papel y las de la versión digital que no fueron impresas. Clarín nombró el término "encuesta" en 30 artículos; Página12, en 43 y La Nación, en 157.

Una vez definido el universo, se sistematizaron las variables y, en esta etapa preliminar de la investigación, se elaboró un libro de códigos que buscaba observar si los diarios 
trataron de manera pareja las encuestas en cada etapa de la campaña, si les asignaron relevancia similar y si utilizaron correctamente las recomendaciones de Esomar y Wapor para la publicación de los sondeos en los medios.

Se busca aplicar las variables definidas para pulir el libro de códigos y poder luego hacer entrevistas en profundidad a editores y redactores. El objetivo final de esta investigación es el de observar qué tan profesionalmente se trata a las encuestas en los medios y, si hay fallas, comprender cuáles son, por qué tienen lugar y cómo podrían subsanarse. Finalmente, se exponen las principales conclusiones surgidas del análisis de esta etapa del estudio.

\subsubsection{Objetivos y preguntas de investigación}

El objetivo general de este trabajo es realizar un análisis exploratorio de la cobertura que los diarios dan a las encuestas en períodos electorales con el propósito de detectar cómo traducen la voz de los ciudadanos para difundirla a su público. Se procura que este trabajo sea validado en estudios posteriores en los que se contrastarán estas respuestas con las que se obtengan en diversas entrevistas en profundidad.

Los objetivos específicos de esta etapa son tres:

- Describir la relevancia que los medios dieron a este asunto en términos de frecuencia de publicación y de jerarquía noticiosa.

- Establecer el comportamiento temporal de dicha relevancia informativa.

- Indagar el tratamiento que los diarios argentinos dan a las encuestas electorales, en especial, en periodos de campaña.

Se buscará detectar si el tratamiento que los medios dan a las encuestas en campañas electorales garantiza que los lectores estén adecuadamente informados sobre lo que significan los datos que brindan los sondeos o si, por el contrario, esos datos se utilizan para reforzar opiniones de periodistas y políticos. 
Este proyecto de investigación nos permitirá observar también si el tratamiento que los medios de comunicación dieron a las encuestas en estas elecciones manifiesta un conocimiento técnico o una intencionalidad por parte de los periodistas.

3.2.2. Etapas y variables formales

El libro de códigos elaborado para el análisis de contenido está estructurado siguiendo las etapas de la campaña electoral (ver Cuadro 2).

Cuadro 2.

Etapas de la campaña

\begin{tabular}{|c|c|}
\hline Fecha & Etapa de análisis \\
\hline $20 / 9$ a $22 / 10$ & Campaña $1^{\mathrm{a}}$ vuelta \\
\hline 22 a $25 / 10$ & Veda $1^{\text {a }}$ vuelta \\
\hline $26 / 10$ a $19 / 11$ & Campaña $2^{\mathrm{a}}$ vuelta \\
\hline 20 a $22 / 11$ & Veda $2^{a}$ vuelta \\
\hline
\end{tabular}

Fuente: Elaboración propia.

Los datos acumulados de un análisis de contenido permiten solo descripciones globales del tipo de cobertura pero no dan razón del porqué de esta. Para ello se requieren pautas de comparación (Amadeo, 1999: 226). En este caso se da lugar a dos ejes de análisis: el primero referido a las diferencias que puede plantear cada medio ante el mismo acontecimiento $\mathrm{y}$, en segundo lugar, la evolución que el discurso pueda sufrir en el transcurso temporal en el caso, para lo cual este fue dividido en etapas.

El objetivo de establecer etapas para el análisis de este caso es doble. Por un lado, analizar cómo evoluciona la cobertura a lo largo del caso y, por otro, observar el comportamiento individual y comparado de los medios con respecto a este tema.

Luego de un primer grupo de variables que buscan describir las características formales de los artículos (título de 
la nota, diario, fecha, sección), siguen otras que apuntan a visualizar la importancia que los medios le dieron al tema (ubicación en el diario, tiene o no imágenes, tamaño, está en tapa o no, se elaboró un cuadro o gráfico a partir de la información estadística o no).

\subsubsection{La cobertura de las encuestas}

Una vez definidas las variables formales y de relevancia, siguen las que analizan hasta qué punto los medios trataron las encuestas de manera rigurosa. La primera variable de análisis observa si junto con los resultados de las encuestas se presentan datos técnicos de alguna naturaleza o no. La segunda destaca, en caso de haber datos técnicos, cómo se presentan estos, si la información técnica está dentro de un recuadro, si se la incorpora al texto o si hay una combinación de ambas opciones.

En cuanto a los datos técnicos en sí, se buscó detectar si las notas reflejan el nombre de la organización de investigación que realizó el sondeo, si hay una o más encuestadoras citadas, si figura el universo efectivamente representado (es decir, quién fue entrevistado), si se menciona el tamaño de la muestra conseguida y su cobertura geográfica, si se indican las fechas del trabajo de campo, si se especifica el método de muestreo utilizado (y en el caso de muestras completamente aleatorias, la tasa de respuesta obtenida), si se aclara el método por el que se recogió la información (cara a cara, entrevista telefónica, panel de internet, etc.), si se aplicó una ponderación para ajustar los resultados, qué universo se utilizó para ello y si se incluyeron las preguntas relevantes.

Para alcanzar nuestro objetivo, proponemos elaborar un libro de códigos cuyo diseño se discute en este trabajo, buscando que esta matriz de análisis pueda ser validada en estudios posteriores que traten la calidad de la cobertura que los medios dan a las encuestas. Este pretest nos permitirá establecer las variables y categorias y la prueba de los indicadores de medida que den cuenta de la forma en que fueron me- 
diáticamente tratadas las encuestas (Piñuel Raigada, 2002), con el propósito de cuantificarlas en un análisis ulterior. ${ }^{4}$

\section{Resultados y discusión}

En esta instancia exponemos las bases sobre las que se sustenta la investigación y desarrollamos la matriz que ha surgido de nuestra aproximación exploratoria al corpus. En ulteriores investigaciones contrastaremos los resultados obtenidos de un análisis cuantitativo deductivo del universo completo con entrevistas en profundidad a los redactores y editores de los periódicos en cuestión.

El libro de códigos elaborado para el análisis de contenido está estructurado en una serie de etapas electorales.

\subsection{Relevancia de la información}

Para comprender la "relevancia" que un medio le otorga a un tema es necesario recurrir a diversos estudios que entienden que para atribuir relevancia a un tema se deben priorizar unos elementos sobre otros y hacerlos más fáciles de recordar (Amadeo, 1999). Para dar una relevancia determinada a las noticias, los periodistas se valen de diversos recursos formales. Lo hacen, principalmente, por medio de la repetición del tema y de la ubicación en la cual presentan la noticia ${ }^{5}$ (ver Cuadro 3).

\section{Cuadro 3. \\ Relevancia de los artículos en los que se nombra encuestas electorales}

\begin{tabular}{ll}
\hline Importancia & Porcentaje \\
\hline Aparece en tapa & $19(\mathrm{~N}=44)$
\end{tabular}

\footnotetext{
${ }^{4}$ Según Piñuel Raigada (2002: 8), estos análisis se dirigen "a resolver los problemas relativos a la elaboración de las categorias más pertinentes que han de configurar un protocolo de análisis y su eventual articulación [...], de modo que resulten productivas de cara a la explotación de los datos".

5 "La 'relevancia' implica visibilidad y recuerdo de la información. Para lograr esto los medios cuentan con la ubicación de la noticia -arriba a la derecha, en página impar-, mayor tamaño, la disposición de la información con respecto a otras o una mayor frecuencia de cobertura del tema" (Amadeo, 2008: 195).
} 


$\begin{array}{ll}\text { Página impar } & 46(\mathrm{~N}=106) \\ \text { Con imagen } & 70(\mathrm{~N}=161) \\ \text { Gran tamaño* } & 51(\mathrm{~N}=117)\end{array}$

Fuente: Elaboración propia.

*Se considera que la nota tiene un "gran tamaño" cuando abarca más de 2/4 de la página de un diario.

Una lectura de estos indicadores en forma desagregada muestra que las notas en las que de un modo u otro se habló de encuestas alcanzó una importancia noticiosa significativa, dado que una de cada cinco unidades fue mencionada en tapa, casi la mitad apareció en página impar, siete de cada diez estuvieron acompañadas de alguna imagen (fotos, dibujos, infografías o caricaturas) y arriba del 50\% ocupó más de la mitad de la página. Ahora bien, los datos acumulados del análisis de contenido permiten solo descripciones globales del tipo de cobertura pero no dan razón del porqué de esta. Para ello se requieren pautas de comparación (Amadeo, 1999: 226). En este caso, el eje de comparación se apoya en la evolución que el discurso pudo haber sufrido en el transcurso temporal del caso, para lo cual este fue dividido en etapas.

Gráfico 1.

Relevancia de la información según momentos del proceso electoral

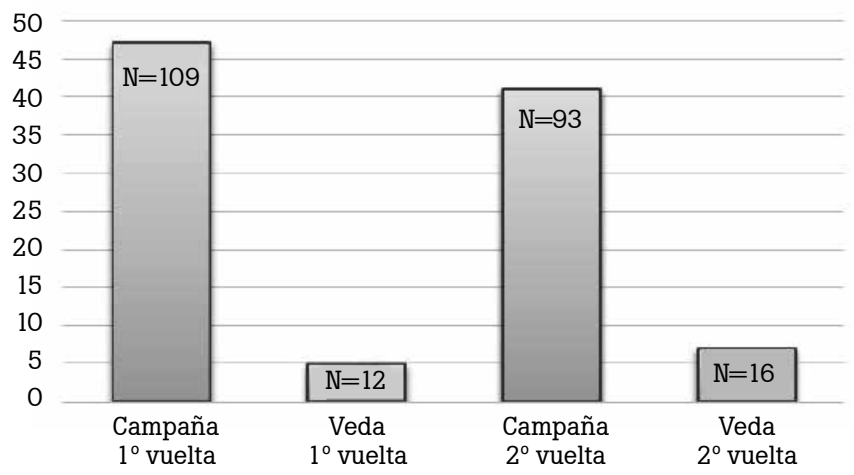

Fuente: Elaboración propia. 
El análisis se dividió en cuatro etapas buscando comprender si hubo diferencias en el tratamiento de las noticias a medida que se desarrollaba el proceso electoral. Los cuatro momentos relevantes en la cobertura fueron 1) la campaña de la primera vuelta electoral, 2) la veda electoral previa a la primera vuelta, 3) la campaña de la segunda vuelta electoral, y 4) la veda electoral previa a la segunda vuelta. Estos hechos marcan etapas dentro del caso, que recibieron coberturas particulares por parte de los medios. Una comparación de estas permite distinguir el grado de relevancia que los medios atribuyeron a la cobertura (ver Gráfico 1).

El gráfico evidencia que las etapas que recibieron mayor cobertura fueros los periodos de campaña, lo cual es comprensible por la extensión en tiempo y por la relevancia del asunto. Es interesante también ver que se nombra las encuestas en los días de veda electoral, previos a las elecciones.

Con respecto a las secciones, la principal sección de los tres diarios analizados es aquella en la que se cubren las elecciones, en algunos casos con subsecciones ad hoc. El $73 \%$ de las notas en las que se mencionan las encuestas fue publicado en esa sección. El restante $27 \%$ fue trabajo de la sección de opinión.

\section{Gráfico 2.}

Porcentaje de notas por sección

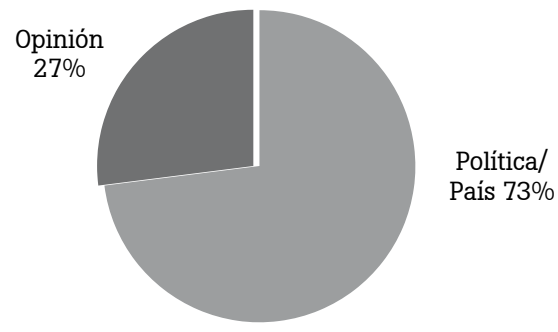

Fuente: Elaboración propia.

\subsection{Tratamiento de las encuestas}

Buscando comprender qué usos les dieron los diarios a las encuestas en la cobertura de las campañas electorales, es 
muy llamativo el dato de qué tipo de mención se hace de ellas. ¿Se trata de artículos dedicados a las encuestas? ¿Son meras menciones destinadas a justificar la opinión del periodista? ¿Son comentarios hechos por políticos?

Un análisis preliminar indica que el 69\% de las notas utilizaron las encuestas como apoyo argumentativo de las opiniones o comentarios realizados por el mismo periodista que escribió la nota, mientras que solo el 14\% del total de las notas relevadas utilizan la encuesta como tema principal, registrándose el $17 \%$ de notas donde las encuestas son mencionadas por algún político a modo de comentario para argumentar o sostener una opinión propia.

\section{Gráfico 3.}

Porcentaje de notas según tipo de cobertura del tema encuestas

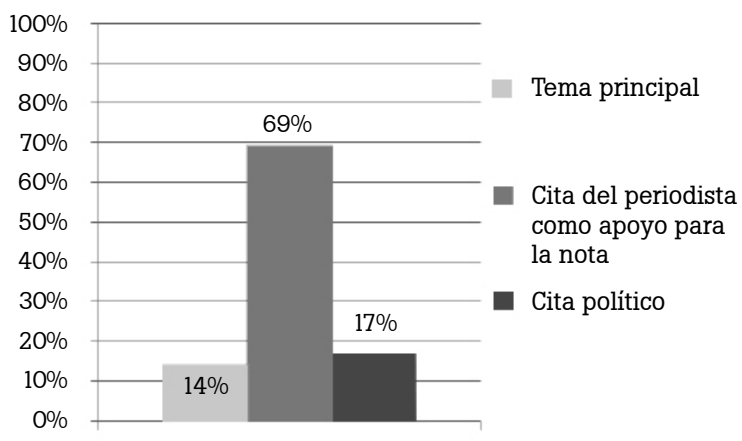

Tipo de cobertura en las encuestas

Fuente: Elaboración propia.

Ante la contundencia de esta información, resultaba interesante comparar también cómo se dio este tratamiento de las encuestas en los diferentes diarios. Siendo que hay un diario oficialista y dos opositores, era posible esperar alguna diferencia ideológica en el manejo de datos; no obstante, una vez analizados los datos es posible afirmar que la cobertura que los diarios hacen de las encuestas es similar, 
sin importar el tamaño de los medios, su impacto en la opinión pública o su tendencia ideológica. Tanto Clarín como Página 12 o La Nación utilizaron las encuestas como apoyo para la argumentación del periodista, registrándose respectivamente los siguientes porcentajes: 57\%, 54\% y 76\% (ver Cuadro 4). En otras palabras, en más de la mitad de las notas los diarios se valieron de encuestas para analizar la coyuntura resaltando opiniones vertidas por el propio periodista.

Cuadro 4.

Porcentaje de notas por tipo de cobertura por medio relevado

\begin{tabular}{|l|c|c|c|}
\hline & Clarín & Página 12 & La Nación \\
\hline Tema principal & $33 \%$ & $23 \%$ & $8 \%$ \\
\hline $\begin{array}{l}\text { Cita del periodista como } \\
\text { apoyo para la nota }\end{array}$ & $57 \%$ & $54 \%$ & $76 \%$ \\
\hline $\begin{array}{l}\text { Cita de político / } \\
\text { entrevistado }\end{array}$ & $10 \%$ & $23 \%$ & $16 \%$ \\
\hline Total & $100 \%$ & $100 \%$ & $100 \%$ \\
\hline
\end{tabular}

Fuente: Elaboración propia.

Llama la atención que los diarios no utilicen más seguido las encuestas como tema principal de sus notas. Ese porcentaje es sensiblemente menor que el de las notas como respaldo para las argumentaciones de los periodistas en los tres diarios relevados y, cuando la encuesta es publicada de manera exhaustiva, el medio la encargó especialmente a una consultora. En el caso particular de La Nación, es interesante destacar el hecho de que a partir de la publicación de la encuesta como nota central, el diario genera ese mismo día y en los días sucesivos una gran cantidad de notas de análisis que se nutren de los datos de esa misma encuesta. Esto no ocurre con la misma claridad en los demás diarios.

En cuanto a la precisión técnica en el tratamiento de las encuestas, los datos que arroja esta investigación muestran que los diarios buscan la argumentación política por sobre 
el rigor técnico. Solo dos de cada diez artículos en los que se mencionan las encuestas muestran datos técnicos (19\%) y en casi todos los casos esos datos están volcados en el texto de la nota (93\%). Un escaso 14\% de las notas del diario La Nación recibe un tratamiento especial en el que la ficha técnica aparece prácticamente completa en un recuadro aparte mientras que los demás diarios no presentan ningún dato técnico fuera de los textos. Solo el 7\% del total de las notas tiene un recuadro de datos técnicos.

\begin{tabular}{|c|c|c|}
\hline \multicolumn{3}{|c|}{$\begin{array}{l}\text { Cuadro } 5 . \\
\text { Porcentaje de artículos que mencionan “encuest } \\
\text { datos técnicos específicos }\end{array}$} \\
\hline \multirow{12}{*}{ Porcentaje d } & Dato técnico específico & $\%$ \\
\hline & Nombre de la consultora & 100 \\
\hline & Universo representado & 11 \\
\hline & Tamaño de la muestra & 34 \\
\hline & Cobertura geográfica & 27 \\
\hline & Fechas del trabajo de campo & 14 \\
\hline & Método de muestreo & 5 \\
\hline & Tipo de encuesta & 18 \\
\hline & Preguntas relevantes & 18 \\
\hline & Error muestral & 5 \\
\hline & Índice de confianza & 5 \\
\hline & $\%$ de NS/NC & 0 \\
\hline
\end{tabular}

Fuente: Elaboración propia.

Otra curiosidad es que sobre la información que se menciona en todos los artículos con datos técnicos es la encuestadora que realizó la investigación. Y el 71\% de las notas que mencionan encuestadoras se refieren a una sola encuestadora, relacionada directamente con el diario en cuestión. Solo tres de cada diez artículos comparan los análisis de distintas encuestadoras.

En el caso de Clarín, la consultora mayormente contratada, según las notas relevadas, fue Management \& Fit, dirigida por Mariel Fornoni, mientras que La Nación publicó de manera exclusiva encuestas realizadas por la consultora Po- 
liarquía, Catterberg y Asociados. Página 12, por su parte, se vincula en mayor grado con la consultora CEOP, conducida por el sociólogo Roberto Bacman, siendo el columnista Raúl Kollmann quien publica este tipo de notas.

El hecho de que un diario contrate regularmente a una $\mathrm{u}$ otra consultora para realizar encuestas exclusivas no implica que dicho medio asuma una postura ideológica en favor o en contra de alguno de los candidatos. A pesar de ello nunca faltan las especulaciones al respecto. Dada la vinculación que habitualmente se tiende a plantear entre las consultoras y los candidatos, los datos aquí relevados no ponen de manifiesto ningún indicio de que exista un tratamiento diferencial en los medios analizados en relación a las encuestas en pos de favorecer o perjudicar a un partido u a otro. Por el contrario, la utilización y mención de datos provenientes de otras consultoras en distintas notas es reiterada en los tres diarios; y hay una tendencia -creemos que involuntaria- a utilizar en muchos casos las mismas fuentes.

En cuanto a la publicación de datos técnicos respetando lo estipulado por Esomar/Wapor, La Nación (14\%) es el único diario que, al publicar una encuesta como nota principal, presenta a sus lectores la ficha técnica -casi completa- en un recuadro separado del texto. Tanto Clarín como Página 12 no solo no lo hacen sino que mencionan muy pocas de las características metodológicas de las encuestas publicadas (fecha del trabajo de campo, cobertura geográfica, tamaño de la muestra y, en menor medida, las preguntas relevantes, el universo de estudio y el método de muestreo y el de recolección de los datos) y en ambos casos lo hace incorporando estos escasos conceptos al mismo texto de la nota. Un dato que no aparece publicado en ninguna de las notas relevadas, independientemente del diario, es el porcentaje de no respuesta de la encuesta, siendo esta una información clave a la hora de interpretar los datos estadísticos. Como señala Beatriz Mañas Ramírez (2008) en su trabajo sobre el tema, no tomar en cuenta cuántos encues- 
tados responden NS/NC es un problema: si los porcentajes de no respuesta son altos, es lícito cuestionar la representatividad de la encuesta.

\section{Gráfico 4.}

Forma de presentación de los datos técnicos en la nota por medio

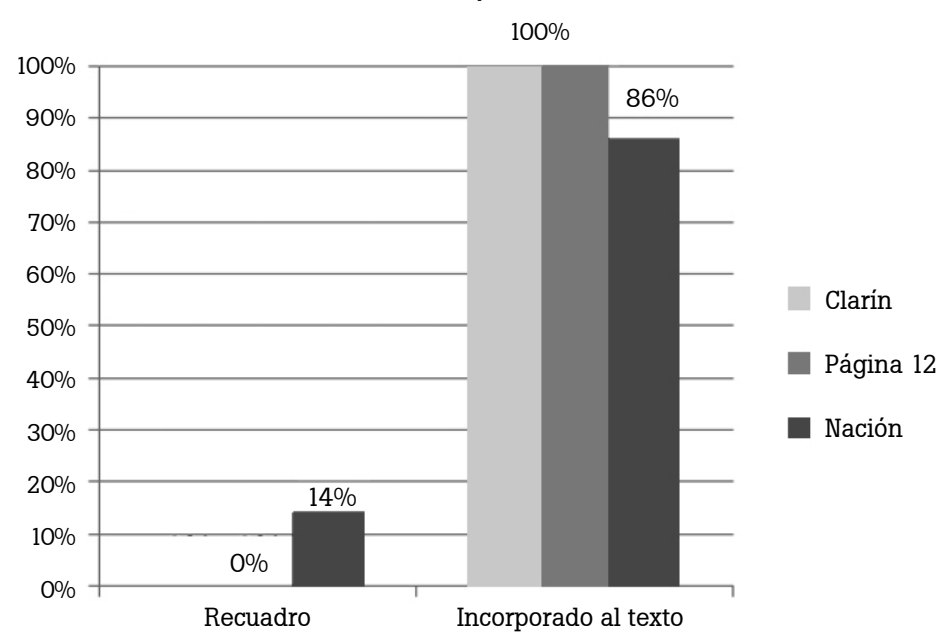

Fuente: Elaboración propia.

\subsection{Conclusiones y reflexiones}

La investigación preliminar sobre la utilización que los diarios hacen de las encuestas cumplió con los objetivos propuestos para esta etapa: se pudo analizar la relevancia que los medios analizados dieron a este asunto en términos de frecuencia de publicación y de jerarquía noticiosa, se pudo establecer el comportamiento temporal de dicha relevancia informativa y se pudo indagar el tratamiento que los diarios argentinos dan a las encuestas electorales en períodos de campaña.

Los datos obtenidos del análisis de contenido de $\mathrm{La} \mathrm{Na-}$ ción, Clarín y Página 12 entre el 20 de septiembre y el 25 de 
noviembre de 2015 permitieron observar que los diarios atribuyeron bastante relevancia a las encuestas durante el período preelectoral. También se vio que las encuestas se utilizaron para fundamentar las ideas de los periodistas y de los candidatos antes que para transmitir información electoral útil a los lectores; en este sentido, las notas periodísticas que cubren las campañas electorales se basan principalmente en reflejar actos y comentarios de los candidatos y no utilizan las encuestas como tema central de análisis objetivo. En la mayor cantidad de oportunidades, las notas de opinión mencionan las encuestas, pero no las analizan de manera técnica.

Es posible afirmar, además, que los diarios no respetan las pautas consensuadas por las asociaciones profesionales internacionales para la difusión de las encuestas, que las encuestas publicadas de manera exhaustiva son hechas exclusivamente para los diarios por consultoras contratadas y que esta situación no se da por decisiones editoriales individuales sino que no hay diferencias sustanciales en el tratamiento de las encuestas por parte de los medios que pueda ser atribuida a posturas ideológicas a favor o en contra del gobierno.

Queda entonces en evidencia que los medios de comunicación se muestran interesados por los datos que las encuestas les brindan a la hora de cubrir las campañas electorales y los diarios argentinos no son una excepción. Durante las campañas electorales argentinas de 2015 esas encuestas fueron principalmente utilizadas para fundamentar análisis de los periodistas o como parte de las declaraciones públicas de los candidatos. Sin casi mostrar fichas técnicas, las encuestas pasan a exhibirse más como un análisis intuitivo de los periodistas que como datos duros que podrían servir como información concreta para ayudar a los ciudadanos a tomar una decisión informada a la hora de votar.

Para mejorar la calidad de la democracia los medios de comunicación deben aportar mucho más que una cobertura de las encuestas que los ayude a argumentar discursivamente su opinión sobre las noticias electorales del día. 
Es fundamental que se instruyan en el uso de las encuestas como herramientas técnicas. Si estas no son bien explicadas, su mala interpretación por parte del público puede llevar a tomar decisiones mal formuladas. Es responsabilidad de los especialistas en estadística aportar a los periodistas todos los datos técnicos necesarios para que realicen un análisis correcto de la situación. Y es obligación primaria de los periodistas intentar comprender bien la importancia de los detalles técnicos de una encuesta.

Los ciudadanos deben tener la posibilidad de poder contar con información fehaciente sobre los datos técnicos de las encuestas y tener todos los elementos necesarios para poder discernir -si así lo desean- sus opiniones y acciones en base a esos datos publicados. En consecuencia, debería ser un deber, tanto de las consultoras como de los medios, brindar a los potenciales electores la información de manera acabada y completa.

Las dos instituciones profesionales de mayor prestigio a nivel internacional se propusieron elaborar la Guía para sondeos de opinión y encuestas publicadas porque este es un problema generalizado. En la Argentina en particular, el nivel de desconocimiento de estas sugerencias técnicas parece ser muy alto en los principales diarios del país. En la próxima instancia de la investigación se preguntará a los periodistas, a los consultores y a los medios cómo es que se forman y se informan sobre estos métodos de medición de la opinión pública.

Los diarios son representativos de los medios y buscan formar a los ciudadanos, acompañarlos en sus decisiones. Cuando contratan consultoras para saber qué opinan esos ciudadanos, no plasman esa voz de manera rigurosa. Sin importar su filiación política, desatienden las precisiones técnicas y validan sus argumentaciones corriendo riesgos de malinterpretar datos de encuestas que les transmiten las consultoras. De este modo, en ocasiones fallan en su objetivo de formar a los ciudadanos para lograr una buena calidad de debate democrático. 


\section{Referencias}

Albornoz, Luis (2006). Periodismo Digital. Los grandes diarios en la red. Buenos Aires: La Crujía.

Alonso, Belén; Daniel Cabrera y María Eugenia Tesio (2009). "Sondeos, votos y voces, aportes para un debate político y técnico". En Maria Braun y C. Straw Opinión Pública: Una mirada desde América Latina. Buenos Aires: Emecé, pp. 173-189.

Amadeo, Belén (1999), La aplicación de la teoría del Framing a la cobertura de la corrupción política en Argentina. 1991-1996. Tesis doctoral inédita. Pamplona, Facultad de Comunicación, Universidad de Navarra, España, 1999.

Amadeo, Belén (2010), "Valores democráticos y medios de comunicación masiva". Anales de la Academia Nacional de Ciencias de Buenos Aires 2008, Academia Nacional de Ciencias de Buenos Aires, Buenos Aires, XLII (1), pp. 283-289, ISBN: 978-987537-108-8.

Amadeo, Belén y Natalia Aruguete (2013). "Medios y Miedos. La cobertura de la inseguridad en la Argentina". Revista de Investigación del Departamento de Humanidades y Ciencias Sociales, Universidad de La Matanza. Año 2- $\mathrm{N}^{\circ} 3$ Mayo 2013, pp. 17-33 ISSN 2250-8139. http://publicaciones.unlam.edu.ar/ rihumso/index.php/humanidades/article/view/27/30.

Amado, Adriana y Maximiliano Bongiovanni (2016). "Periodismos argentinos: perfiles y contextos". En Adriana Amado et al., Periodismos argentinos: modelos y tensiones del siglo XXI, Buenos Aires: Konrad Adenauer Stiftung, pp. 123-153.

Aruguete, Natalia y Belén Amadeo (2013). "Encuadrando el delito: pánico moral en los periódicos argentinos", América Latina Hoy, Revista de Ciencias Sociales, Universidad de Salamanca, $\mathrm{N}^{\circ}$ 62: 177-196, enero de 2013. ISSN: 1130-2887. http://campus.usal.es/ revistas_trabajo/index.php/1130-2887/article/ view/9350/9655.

Becerra, Martín y Guillermo Mastrini (2009), Los dueños de la palabra. Acceso, estructura y concentración de los medios en la América Latina del siglo XXI. Buenos Aires: Prometeo.

Esomar/Wapor (2009). Guía para sondeos de opinión y encuestas publicadas. Obtenido de https://www.esomar.org/uploads/ 
public/knowledge-and-standards/codes-and-guidelines/ ESOMAR_Guia-WAPOR-para-sondeos-de-opinion-y-encuestaspublicadas.pdf.

Igartua, Juan José (2006). Métodos cuantitativos de investigación en comunicación, Barcelona: Bosch.

Mañas Ramirez, Beatriz (2008). "La medida de la Opinión Pública. Una mirada sociológica". En Gonzalo Capellán de Miguel (ed.), Opinión Pública, historia y presente. Madrid: Trotta. pp. 159179.

Miller, Mark M. y Parnell B. Riechert (2001). "The Spiral of Opportunity and Frame Resonance. Mapping the Issue Cycle in News and Public Discourse". En S. D. Reese, O. H. Gandy y A. E. Grant (eds.), Framing public life. Perspectives on media and our understanding of the social world (pp. 107-121). Mahwah, NJ: Lawrence Erlbaum Associates.

Monzón, C. y J. L. Dader (1992). "Las encuestas y su tratamiento periodístico". En Alejandro Muñoz Alonso et al., Opinión pública y comunicación política. Madrid: Eudema, pp. 465-487.

Piñuel Raigada, José Luis (2002). "Epistemología, metodología y técnicas del análisis de contenido", Estudios de Sociolingüística 3(1), 2002, pp. 1-42.

Traugott, M. W. (1997). Encuestas: guía para lectores. México: Siglo XXI.

Tevélez, F. y V. González (2014). "Uso de las encuestas por parte de los diarios en un contexto electoral". Investigación no publicada. Programa Grupos de Investigación en Ciencia Politica Opinión pública, comunicación política y elecciones legislativas. Argentina 2013. Buenos Aires, Carrera de Ciencia Política, Facultad de Ciencias Sociales, Universidad de Buenos Aires.

Ulanovsky, Carlos (1997). Paren las rotativas. Historia de los grandes diarios, revistas y periodistas argentinos. Buenos Aires: Espasa Calpe Argentina.

Vommaro, Gabriel (2008). "Los usos políticos y periodísticos de las encuestas de opinión: entre la sociedad real y la representación del hombre común". Lo que quiere la gente. Sondeos de opinión y el espacio de la comunicación política argentina. Buenos Aires: Prometeo, pp. 119-162. 
Wolton, Dominique (1998). "La comunicación política: la construcción de un modelo". En G. A. Gauthier, Gosselin y J. Mouchon (comp.), Comunicación y política, Barcelona: Gedisa editorial. 